\title{
A simple strategy to effectively produce D-lactate in crude glycerol-utilizing Escherichia coli
}

Yao-De Wang ${ }^{1}$, Jin-Yi Liao', Chung-Jen Chiang ${ }^{2^{*}}$ and Yun-Peng Chao ${ }^{1,3,4^{*}}$

\begin{abstract}
Background: Fed-batch fermentation has been conventionally implemented for the production of lactic acid with a high titer and high productivity. However, its operation needs a complicated control which increases the production cost.

Results: This issue was addressed by simplifying the production scheme. Escherichia coli was manipulated for its glycerol dissimilation and D-lactate synthesis pathways and then subjected to adaptive evolution under high crude glycerol. Batch fermentation in the two-stage mode was performed by controlling the dissolved oxygen (DO), and the evolved strain deprived of poxB enabled production of $100 \mathrm{~g} / \mathrm{L}$ D-lactate with productivity of $1.85 \mathrm{~g} / \mathrm{L} / \mathrm{h}$. To increase productivity, the producer strain was further evolved to improve its growth rate on crude glycerol. The fermentation was performed to undergo the aerobic growth with low substrate, followed by the anaerobic production with high substrate. Moreover, the intracellular redox of the strain was balanced by fulfillment of the anaerobic respiratory chain with nitrate reduction. Without controlling the $\mathrm{DO}$, the microbial fermentation resulted in the homofermentative production of D-lactate (ca. $0.97 \mathrm{~g} / \mathrm{g}$ ) with a titer of $115 \mathrm{~g} / \mathrm{L}$ and productivity of $3.29 \mathrm{~g} / \mathrm{L} / \mathrm{h}$.

Conclusions: The proposed fermentation strategy achieves the highest yield based on crude glycerol and a comparable titer and productivity as compared to the approach by fed-batch fermentation. It holds a promise to sustain the continued development of the crude glycerol-based biorefinery.
\end{abstract}

Keywords: Batch fermentation, Metabolic engineering, Crude glycerol, D-Lactate

\section{Background}

The concern over environmental pollutions caused by petrochemicals-derived materials has urged a pressing need for biopolymers. Recognized as an eco-friendly material, polylactide (PLA) made from D- or L-lactic acid is featured with biocompatibility and processability [1]. PLA naturally hydrolyzes to the non-toxic constituent of $\alpha$-hydroxy acid, which renders it appealing for biomedical applications [2]. Moreover, PLA can be processed in a mold due to its thermoplastic nature. Products of molded

\footnotetext{
*Correspondence: oleosin91@yahoo.com.tw; ypchao@fcu.edu.tw ${ }^{1}$ Department of Chemical Engineering, Feng Chia University, 100 Wenhwa Road, Taichung 40724, Taiwan

2 Department of Medical Laboratory Science and Biotechnology, China Medical University, No. 91, Hsueh-Shih Road, Taichung 40402, Taiwan Full list of author information is available at the end of the article
}

PLA are proved to be non-hazardous by the United State Food and Drug Administration and applicable for food packaging [3]. In particular, the stereocomplex PLA composed of poly D- and L-lactic acid displays a superior performance in terms of thermal stability and mechanical strength [4]. It has a potential application in the electronic sector and the automobile industry [5].

In addition to the synthesis of PLA, lactic acids of enantiomerical purity are required for the application in the textile, food, pharmaceuticals, and chemicals industries [6]. The chemical synthesis of lactic acid lies in the use of petrochemical resources and produces a racemic mixture containing $\mathrm{D}$ - and L-lactic acid. In contrast, the fermentation process implementing a producer strain with the desired trait enables the production of an optically pure lactic acid. The demand 
for L-lactic acid in the food industry has been historically fulfilled by performing the fermentation process with lactic acid bacteria (LAB) [3]. LAB are a diverse group of natural producer and capable of producing lactic acid with a high yield and high productivity. The LAB-based fermentation is conducted mainly with the glucose-rich feedstock [7].

The current price of PLA is not competitive with that of the petroleum-based plastics. To lower the production cost, the producer strain has to grow on a cost-effective medium and synthesize lactic acids with a titer $>100 \mathrm{~g} / \mathrm{L}$, a conversion yield close to the theoretical value, an optical purity $>99 \%$, and high productivity [8]. The efficiency of a lactic acid-based production scheme closely links to the producer strain, the culture substrate, and the operational mode [6]. The growth of LAB generally requires a supplement of complex nutrients. Therefore, many studies have investigated a potential of surrogate bacteria involving Bacillus sp. [9], Escherichia coli [10], and Corynebacterium glutamicum [11]. Reported results for these strains receiving the genetic modification are generally encouraging. The lactic acid fermentation has been commonly conducted with glucose. However, the replacement of glucose with a renewable substrate reduces the production cost. Fed-batch fermentation circumvents the problem of substrate inhibition and enhances the production titer. The feeding strategy by maintaining glucose at a certain level was illustrated as an effective method for the production of lactic acids [12]. Nevertheless, it requires more labors and equipments to operate.

Crude glycerol appears to be a renewable resource which attracts the industrial attentions [13]. It is a byproduct in the waste stream resulting from the production process of biodiesel. Social problems associated with the intensive use of fossil fuels have increased a demand for biodiesel. The refinement of crude glycerol for the production of value-added chemicals provides an appealing way to fulfill circular economy in the biodiesel industry [14]. In view of immaturity of the industrial fermentation process for D-lactic acid, we previously worked on the crude glycerol-based production of D-lactic acid in E. coli. The producer strain was obtained by manipulating its metabolic pathways leading to D-lactic acid. By fed-batch fermentation, the engineered strain enabled high production of D-lactic acid (>99\% optical purity) [15]. In this study, our continued effort was made to simplify the operational mode. A simple strategy based on batch fermentation was developed for the effective production of D-lactate from crude glycerol.

\section{Results and discussion}

\section{Development of producer strain}

In our previous work, fed-batch fermentation of strain BLac-2106 was illustrated to effectively produce D-lactate using crude glycerol in terms of a high titer and high productivity [15]. In essence, this strain was deficient in $a d h E$, frdA, pflB, and pta to curtail the carbon waste for the synthesis of byproducts including ethanol, formate, succinate, and acetate (Fig. 1). To enhance the synthesis of D-lactate, the strain was equipped with a genomic copy of the $\lambda \mathrm{P}_{\mathrm{L}}$ promoter $\left(\mathrm{P} \lambda \mathrm{P}_{\mathrm{L}}\right)$-driven $\mathrm{D}$-ldh from Lactobacillus helveticus at pflCD. The strain was also deprived of $d l d$ and $m g s A$, which renders it unable to metabolize the enantiomerical purity of $\mathrm{D}$-lactate. Although the fermentation result is encouraging, the fed-batch mode is complicated and unfriendly to operate. Therefore, this study was aimed to simplify the production scheme. As indicated in Fig. 1, the synthetic route leading to D-lactate from glycerol generally contains 3 module pathways participating in glycerol dissimilation, the supply of pyruvate precursor and energy, and the D-lactate synthesis. The dissimilation and synthesis pathways were reasoned to be limited. Therefore, strain BLac-2106 was further modified by genomic fusion of $\mathrm{P} \lambda \mathrm{P}_{\mathrm{L}}$ with endogenous gldA, dhaKLM, and $l d h A$. The latter encodes lactate dehydrogenase (LDH) responsible for the conversion of pyruvate into D-lactate [16]. The catalytic function of GldA and DhaKLM mediates the fermentative dissimilation of glycerol [17]. The modified strain displayed a $40 \%$ increase in the LDH. The expression level of gldA and of dhaKLM in the strain was elevated by $45 \%$ and $80 \%$, respectively. The implementation of batch fermentation to achieve a high titer is challenging because of substrate inhibition. The modified strain was, thus, subjected to the adaptive evolution, and one resulting strain (designated EcoB140) was scored for further characterization (Additional file 1: Figure S1).

\section{Production of $\mathrm{D}$-lactate by batch fermentation}

Batch fermentation in the two-stage mode was conducted with strain EcoB-140 using crude glycerol $(110 \mathrm{~g} / \mathrm{L})$. This was first performed with high aeration for rapid accumulation of cell biomass (i.e. the growth phase). Meanwhile, yeast extract $(10 \mathrm{~g} / \mathrm{L})$ was supplemented to eliminate the lag growth of the strain. After the termination of bacterial growth at $12 \mathrm{~h}$ of the fermentation, the production phase was initiated simply by lowering the dissolved oxygen (DO) tension to $5 \%$ of the saturated level. At the end of the fermentation, the strain produced D-lactate of $70 \mathrm{~g} / \mathrm{L}$ at the expense of $90 \mathrm{~g} / \mathrm{L}$ crude glycerol (Fig. 2a). A large portion of $\mathrm{D}$-lactate appeared in the production phase. 


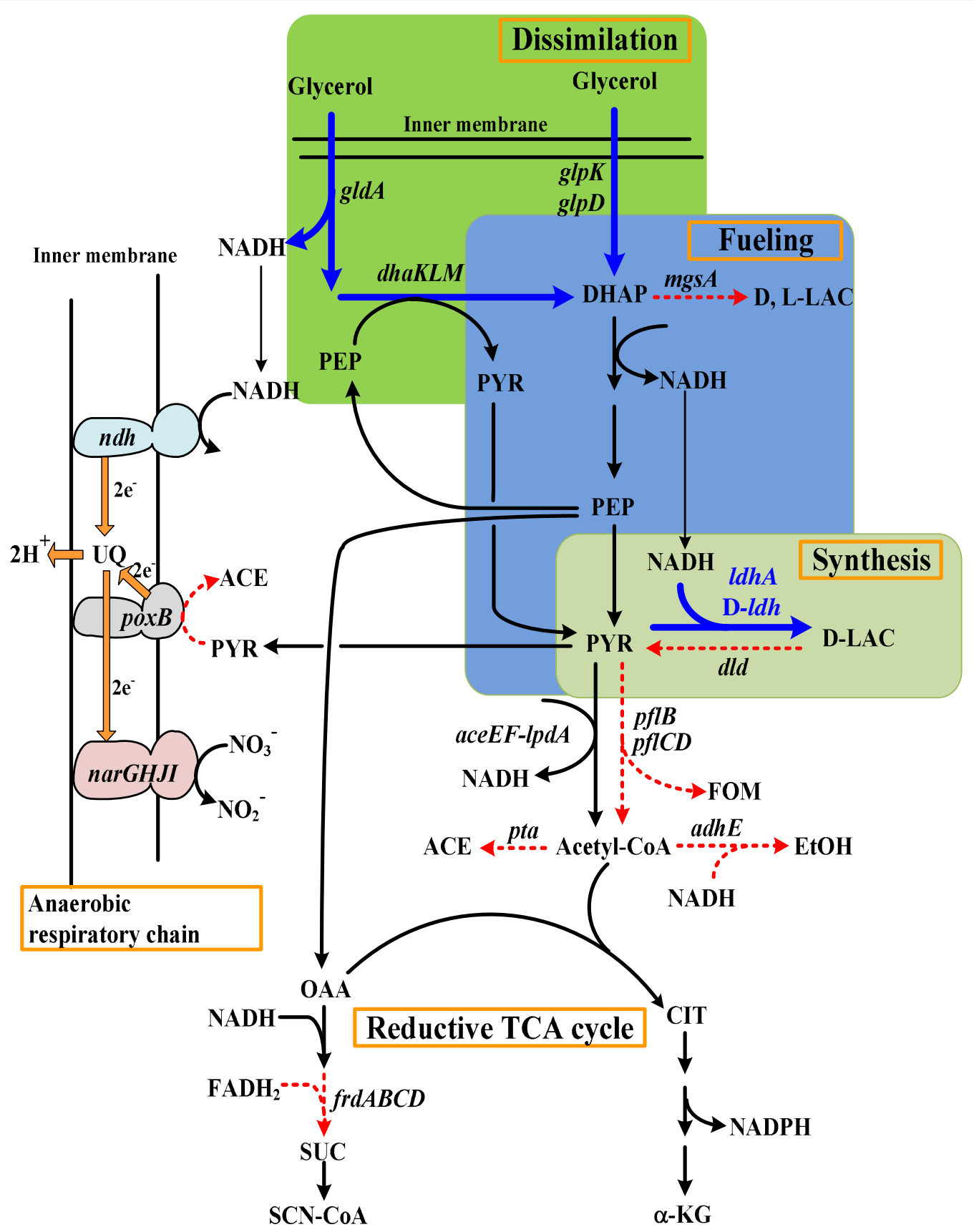

Fig. 1 Metabolic pathways of interest leading to the synthesis of D-lactate from glycerol. The enhanced and blocked pathways were highlighted in blue (solid line) and red (dotted line), respectively. Genes and key metabolites involved in metabolic pathways include as follows: aceE-Ipd, pyruvate dehydrogenase; adhE, aldehyde-alcohol dehydrogenase; dld, FAD-linked D-lactate dehydrogenase; dhaKLM, dihydroxyacetone kinase operon; frdABCD, fumarate reductase operon; IdhA, NAD-linked D-lactate dehydrogenase; D-Idh, L. helveticus D-lactate dehydrogenase; $m g s A$, methylglyoxal synthase; $n$ arGHJl, nitrate reductase operon; $n d h$, NADH dehydrogenase; $p o x B$, pyruvate oxidase; gldA, glycerol dehydrogenase; glpD, glycerol 3-phosphate dehydrogenase; glpK, glycerol kinase; $f$ IB, pyruvate-formate lyase; $p f l D C$, putative pyruvate-formate lyase; pta, phosphate acetyltransferase; ACE acetate, CIT citrate, DHAP dihydroxyacetone phosphate, EtOH ethanol, FOM formate, a-KG a-ketoglutarate, D-LAC D-lactate, L-LAC L-lactate, OAA oxaloacetate, PEP phosphoenolpyruvate, PYR pyruvate, SUC succinate, SCN-CoA succinyl-CoA, UQ ubiquinone

It was unexpected to detect the production of acetate for the strain grown on a high concentration of glycerol (Fig. 2a). Our previous study illustrated the implementation of fed-batch fermentation for the production of D-lactate with negligible byproducts [15]. This was carried out by a stepwise feeding of glycerol to keep 

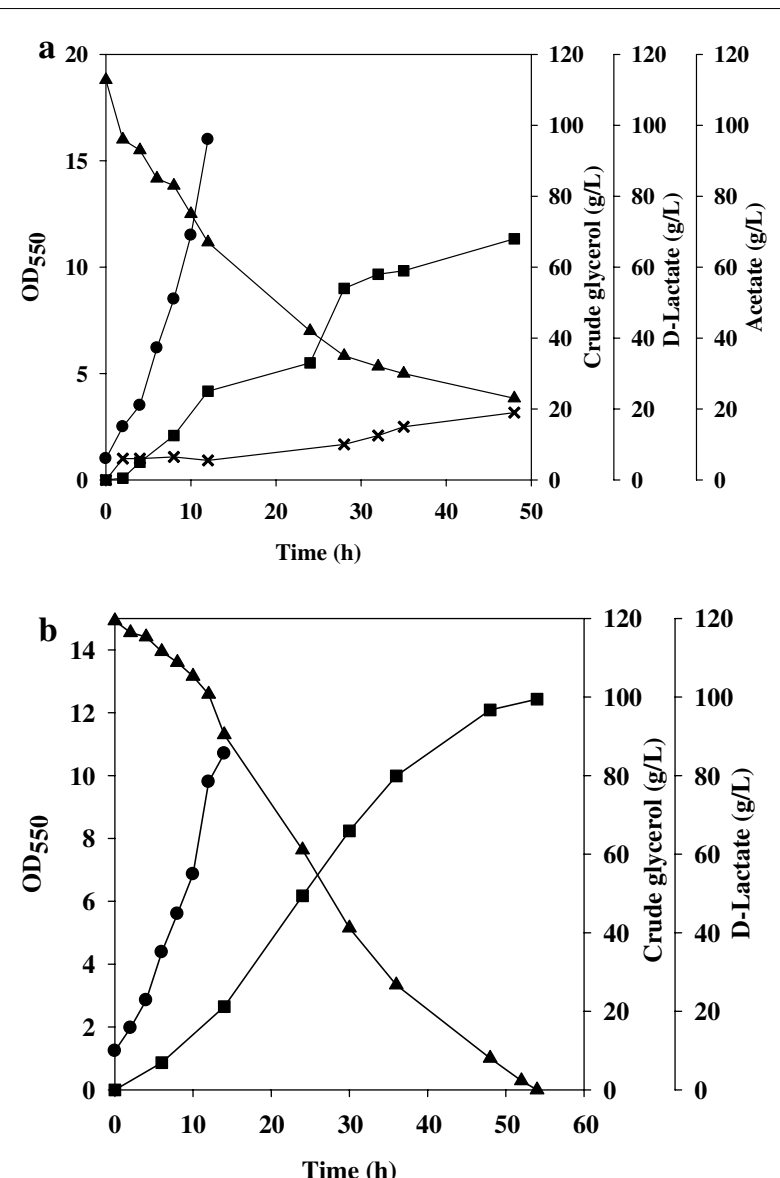

Fig. 2 Production of D-lactate by batch fermentation. The engineered strain was cultured in a bench fermenter containing crude glycerol. The batch fermentation was under the control of the DO to proceed the aerobic growth phase and the fermentative production phase. The fermentation was initiated with the cell density at $\mathrm{OD}_{550}$ of 1 . The absorbance measurement for the cell density in the production phase was interfered with crude glycerol and $\mathrm{Ca}(\mathrm{OH})_{2}$ and, therefore, not reported. The experiment was duplicated. The typical fermentation profiles were reported for strain EcoB-140 (a) and strain EcoB-140B (b). Cell density (black circle); crude glycerol (black up-pointing triangle); D-lactate (black square); acetate $(X)$

its level below $10 \mathrm{~g} / \mathrm{L}$ during the fermentation process. Overall, acetate overflow likely correlates with the glycerol level. Pyruvate oxidase encoded by poxB in E. coli catalyzes the oxidative decarboxylation of pyruvate to acetate, and its expression level is induced in the presence of glycerol and in response to osmotic stress as well [18]. Accordingly, a high expression level of poxB could be realized in the strain exposed to a high concentration of glycerol. The absence of acetate in the glycerol-grown E. coli was due to the reutilization of acetate mediated by acs-encoded acetyl-CoA synthetase [18]. Acetate overflow occurring in strain EcoB-140 is likely the result of the synthesis rate exceeding the utilization rate. Accordingly, strain EcoB-140 was deprived of poxB to give strain EcoB-140B. Batch fermentation of the resulting strain was carried out with crude glycerol $(120 \mathrm{~g} / \mathrm{L})$ and yeast extract $(10 \mathrm{~g} / \mathrm{L})$. As shown in Fig. 2b, strain EcoB-140B consumed around $30 \mathrm{~g} / \mathrm{L}$ glycerol in the first $12 \mathrm{~h}$ of the fermentation (i.e., the growth phase). In the production phase, D-lactate started to largely accumulate and reached around $100 \mathrm{~g} / \mathrm{L}$ at $53 \mathrm{~h}$ of the fermentation. Acetate was not detected at the end of the fermentation.

\section{Production of $\mathrm{D}$-lactate by the two-dose fermentation}

Crude glycerol drastically perturbs the physiological status of microbes [19]. It appears that the engineered strain is susceptible to the stress incurred by a high concentration of crude glycerol, which in turn retards the bacterial growth and decreases productivity. This issue may be addressed by the approach using a high cell density. Batch fermentation with two substrate doses (i.e., two-dose fermentation) was, thus, performed by growing the strain on a low dose of crude glycerol, followed by adding a high dose to the culture upon initiation of the production phase. The strain was first cultured with high aeration in the presence of crude glycerol $(30 \mathrm{~g} / \mathrm{L})$ plus yeast extract $(2 \mathrm{~g} / \mathrm{L})$. As shown in Fig. 3a, the strain almost consumed all glycerol at $9 \mathrm{~h}$ of the fermentation. Subsequently, crude glycerol of $70 \mathrm{~g} / \mathrm{L}$ was added into the fermentation broth and the DO was controlled at around $5 \%$ of the saturated level. At $30 \mathrm{~h}$ of the fermentation, the strain consumed all glycerol and produced D-lactate of $92 \mathrm{~g} / \mathrm{L}$. The result indicates that this proposed strategy has a potential to improve the strain's performance on crude glycerol in terms of productivity (from 1.85 to $3.1 \mathrm{~g} / \mathrm{L} / \mathrm{h}$ ).

Unexpectedly, the fermentation ended with the associated production of $\alpha$-ketoglutarate $(\alpha-K G)$ in the strain (Fig. 3a). The tricarboxylic acid (TCA) cycle proceeds to the reductive pathway consisting of two terminal nodes at succinyl-CoA and $\alpha-K G$ in fermenting E. coli (Fig. 1) [20]. The lack of frdA (encoding fumarate reductase) conserves oxaloacetate (OAA) in strain EcoB-140B, which in turn favors the catalytic conversion of available OAA with acetyl-CoA to citrate. Citrate is further oxidized to $\alpha-K G$. It is recognized that the low oxygen tension negates the functional activity of the TCA cycle [21]. In the absence of oxygen, E. coli relies on either nitrate reductase or fumarate reductase to reduce nitrate or fumarate which serves as the terminal electron acceptor in the respiratory chain [22]. Accordingly, the production phase was conducted under the low oxygen tension. To further simplify the operation, the fermentation was carried out without controlling the DO. The strain was cultured on crude glycerol $(30 \mathrm{~g} / \mathrm{L})$ plus yeast extract $(2 \mathrm{~g} / \mathrm{L})$ for $9 \mathrm{~h}$ with a high agitation at $500 \mathrm{rpm}$ in the growth 

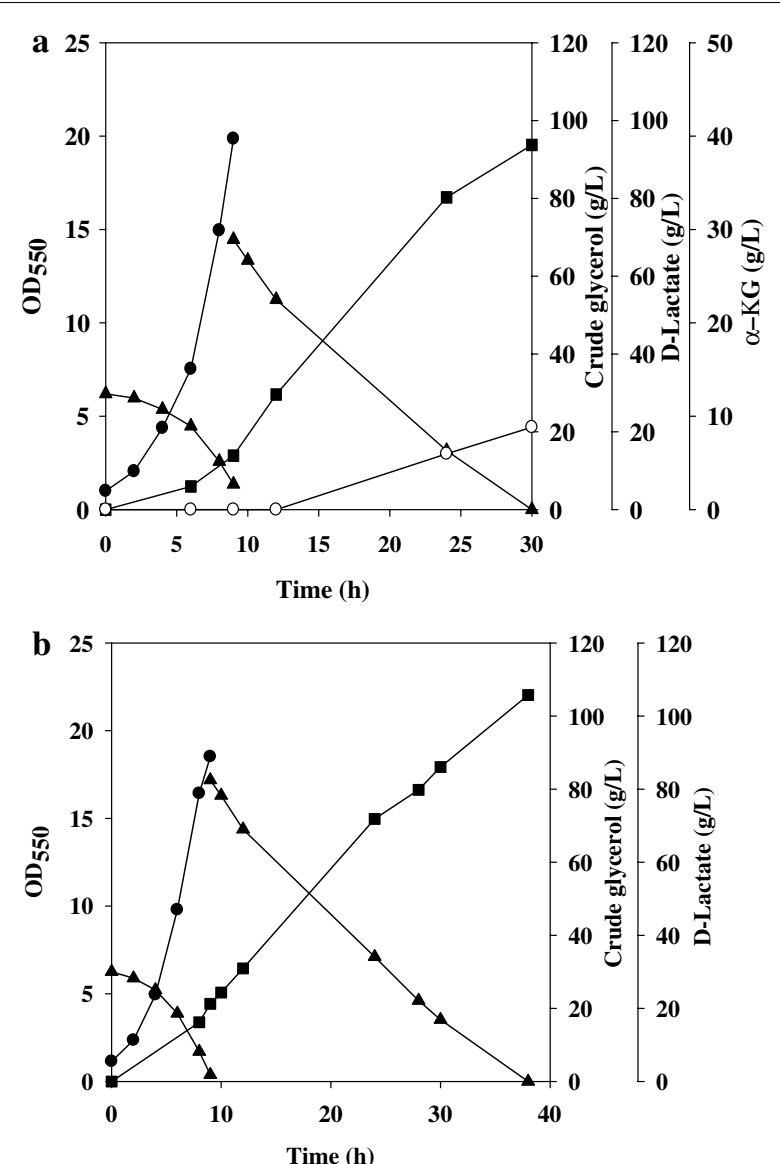

Fig. 3 Production of $\mathrm{D}$-lactate by the two-dose fermentation. The fermentation was conducted with the aerobic growth phase and the fermentative production phase. A high dose of crude glycerol was added to the culture broth at the end of the growth phase. The fermentation was initiated with the cell density at $\mathrm{OD}_{550}$ of 1 . The absorbance measurement of the cell density in the production phase was interfered with crude glycerol and $\mathrm{Ca}(\mathrm{OH})_{2}$ and, therefore, not reported. The experiment was duplicated. The typical fermentation profiles were reported for the cases with the DO control (a) and without the DO control (b). Cell density (black circle); crude glycerol (black up-pointing triangle); D-lactate (black square); a-KG (white circle)

phase. In the second phase, crude glycerol $(80 \mathrm{~g} / \mathrm{L})$ and $\mathrm{NH}_{4} \mathrm{NO}_{3}(20 \mathrm{mM})$ were added to the culture broth and the fermentation proceeded to the production of D-lactate with a low agitation at $200 \mathrm{rpm}$. It was found that the DO fluctuated below $1 \%$ of the saturated level. The strain consumed all glycerol and produced $105 \mathrm{~g} / \mathrm{L}$ D-lactate at $38 \mathrm{~h}$ of the fermentation (Fig. 3b). $\alpha-K G$ was not detected at the end of the fermentation. Nitrate reductase is expressed in response to nitrate and fulfills its role in the respiratory chain of fermenting bacteria. As a result, it provides an electron sink to balance the intracellular redox level (Fig. 1). Note that NADH dehydrogenase encoded by $n d h$ primarily functions in the nitrate respiration [23].

\section{Effective production of $\mathrm{D}$-lactate}

The poxB-deficient strain indeed displayed a slower growth rate, in agreement with the growth defect of the mutant strain on glucose [24]. The PoxB-mediated reaction is coupled to the respiratory chain via ubiquinone and generates the proton motive force [25]. The strain without PoxB is likely in the shortage of energy. To improve its growth, strain EcoB-140B was evolved in the presence of $30 \mathrm{~g} / \mathrm{L}$ crude glycerol without yeast extract. Finally, one strain (designated EcoB-170) displaying better growth was scored for investigation. The two-dose fermentation of strain EcoB-170 was carried out as described earlier except that the initial cell density (i.e., 0.2 at $\mathrm{OD}_{550}$ ) was reduced to one fifty of that used in Fig. 3b. As a result, the strain solely produced D-lactate with a titer of $105 \mathrm{~g} / \mathrm{L}$ at $30 \mathrm{~h}$ of the fermentation (Fig. 4a). The performance of strain EcoB-170 was further investigated for its growth on a lower level of yeast extract (i.e., $1 \mathrm{~g} / \mathrm{L}$ ). The production phase was initiated with a higher substrate dose (i.e., $90 \mathrm{~g} / \mathrm{L}$ ). It consequently resulted in D-lactate of $115 \mathrm{~g} / \mathrm{L}$ at $35 \mathrm{~h}$ of the fermentation (Fig. 4b).

The fermentative production of D-lactate has been mostly conducted with glucose. There are few studies reporting the use of crude glycerol. In one report, $E$. coli strain B0013-070 was constructed with overexpression of $l d h A$ and the deletion of ackA-pta, adhE, dld, frdA, pflB, poxB, and pps [26]. The fermentation was conducted with the aerobic phase at $34{ }^{\circ} \mathrm{C}$, followed by the microaerobic phase at $40{ }^{\circ} \mathrm{C}$. The $\mathrm{DO}$ was controlled below $1 \%$ of the saturated level and the glycerol level was maintained above $10 \mathrm{~g} / \mathrm{L}$ during the microaerobic phase. As a result, the strain produced D-lactate of $100 \mathrm{~g} / \mathrm{L}$ with the conversion yield and productivity reaching $0.75 \mathrm{~g} / \mathrm{g}$ and $2.78 \mathrm{~g} / \mathrm{L} / \mathrm{h}$, respectively (Table 1 ). Our previous work reported the development of strain BLac-2106 by genetic manipulation of the central metabolism, particularly the glycerol catabolism [15]. The production phase was proceeded to maintain the DO at $1 \%$ of the saturated level by adjusting the feeding dose of glycerol after the termination of the growth phase. The implementation of fed-batch fermentation enabled the engineered strain to produce $\mathrm{D}$-lactate with a titer of $105 \mathrm{~g} / \mathrm{L}$, a yield of $0.75 \mathrm{~g} / \mathrm{g}$, and productivity of $2.78 \mathrm{~g} / \mathrm{L} / \mathrm{h}$. Others with the emphasis on pure glycerol have been also reported. One study was conducted with the thiE-null strain B0013-080A which was unable to synthesize thiamine monophosphate [27]. The growth of strain B0013-080A was controlled by the availability of supplemented thiamine, and D-lactate was produced 

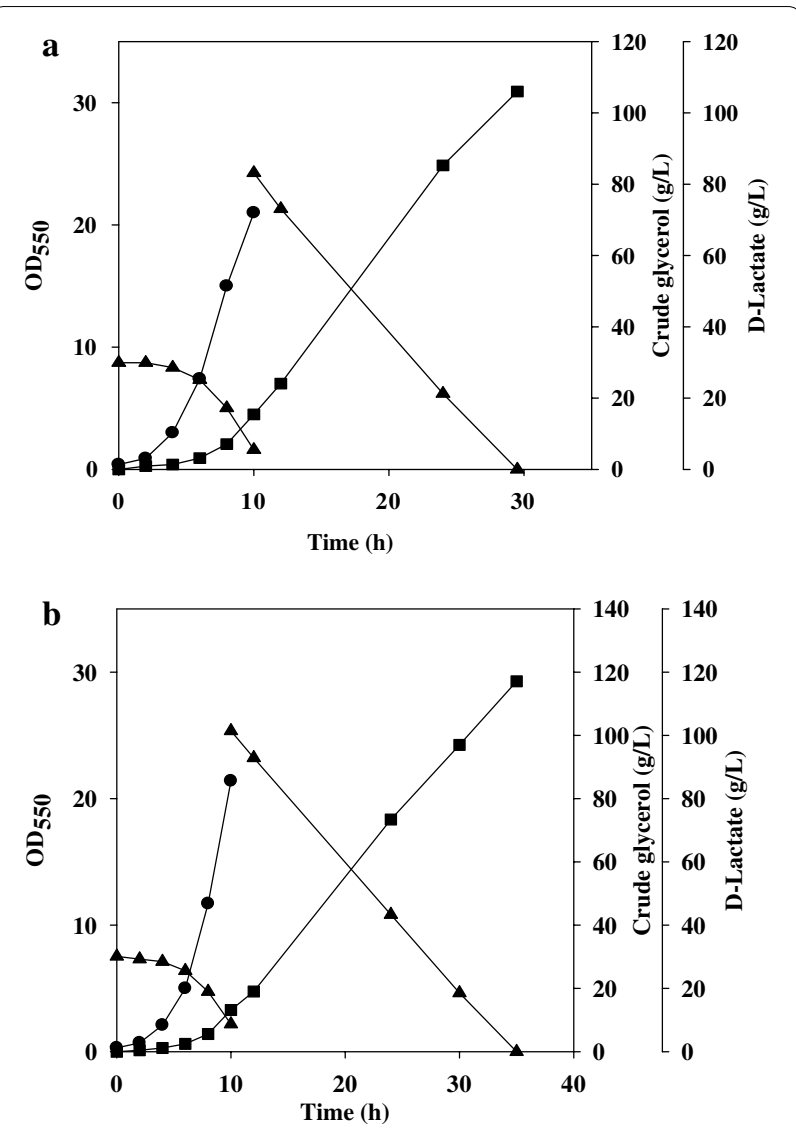

Fig. 4 Effective production of D-lactate by the two-dose fermentation. Refer to Fig. 3 for the fermentation process. The fermentation was initiated with the cell density at $\mathrm{OD}_{550}$ of 0.2 . The absorbance measurement of the cell density in the production phase was interfered with crude glycerol and $\mathrm{Ca}(\mathrm{OH})_{2}$ and, therefore, not reported. The experiment was duplicated. The typical fermentation profiles were reported for strain EcoB-170 (a) grown on $2 \mathrm{~g} / \mathrm{L}$ yeast extract and fed with $80 \mathrm{~g} / \mathrm{L}$ crude glycerol and (b) grown on $1 \mathrm{~g} / \mathrm{L}$ yeast extract and fed with $90 \mathrm{~g} / \mathrm{L}$ crude glycerol. Cell density (black circle); crude glycerol (black up-pointing triangle); D-lactate (black square)

for the non-growing strain upon the depletion of thiamine. Fed-batch fermentation was carried out by dosing 4 batches of glycerol, and the strain produced D-lactate with a titer of $120 \mathrm{~g} / \mathrm{L}$, a yield of $0.87 \mathrm{~g} / \mathrm{g}$, and productivity of $4.11 \mathrm{~g} / \mathrm{L} / \mathrm{h}$. Another work reported the isolation of $E$. coli strain AC-521 from soil samples. By keeping glycerol between 20 and $30 \mathrm{~g} / \mathrm{L}$, the fermentation of the strain in a fed-batch mode led to the production of lactic acid of $86 \mathrm{~g} / \mathrm{L}$ at $40{ }^{\circ} \mathrm{C}$ [28]. The result accounts for a yield of $0.88 \mathrm{~g} / \mathrm{g}$ and productivity of $0.97 \mathrm{~g} / \mathrm{L} / \mathrm{h}$. In addition, Klebsiella pneumoniae was deprived of $d h a T$ and $y q h D$ to block the synthetic pathway of 1,3-propanediol [29]. Glycerol was continuously fed at a constant rate in the microaerobic phase. By fed-batch fermentation, the strain with the overexpression of $l d h A$ enabled production of $142 \mathrm{~g} / \mathrm{L}$ D-lactate with a yield of $0.82 \mathrm{~g} / \mathrm{g}$ and productivity of $2.96 \mathrm{~g} / \mathrm{L} / \mathrm{h}$. Nevertheless, the fermentation with $K$. pneumoniae requires the supplement of peptone and beef extract.

The complication of controlling the substrate level in a bioreactor makes fed-batch fermentation practically ineffective. Moreover, the operation usually needs sophisticated devices which increase the equipment investment. Batch fermentation apparently simplifies the production process without these drawbacks. However, the problem of substrate inhibition presents to be challenging. The brewing industry has implemented the very high gravity fermentation (VGHF) technology to remain profitable [30]. Ethanolic yeast is afflicted with the disorder in membrane fluidity and glucose metabolism under the hypertonic environment originating from VGHF [31]. The cellular utilization of glucose is retarded by the decreased expression of certain glycolytic enzymes [32]. A defect in membrane fluidity handicaps the solute transport systems of cells [33]. In contrast, a high level of crude glycerol induces the inhibitory effect on $E$. coli and the underlying mechanism may vary with the various compositions of crude glycerol. The producer strain was, thus, evolved in the hypertonic solution of crude glycerol. The supplement of yeast extract helped the cell growth in batch fermentation, whereas acetate overflow occurred for the evolved strain (Fig. 2a). This issue was addressed by deletion of poxB in the strain. The resulting strain enabled production of $100 \mathrm{~g} / \mathrm{L}$ D-lactate with a yield of $0.95 \mathrm{~g} / \mathrm{g}$ and productivity of $1.85 \mathrm{~g} / \mathrm{L} / \mathrm{h}$ (Table 1 ). To improve productivity, the strain was further evolved (i.e., strain EcoB-170) and was then employed for the twodose fermentation. This process was first conducted with a light dose in the bioreactor to favor the aerobic growth. In the second stage, a heavy dose was added to speed up the anaerobic catabolism of glycerol. Note that this approach leads to a drastic reduction in yeast extract and initial cell density as applied for the microbial fermentation, which lowers the production cost. Consequently, strain EcoB-170 produced the optically pure D-lactate (>99\%) with a titer of $115 \mathrm{~g} / \mathrm{L}$, a yield of $0.97 \mathrm{~g} / \mathrm{g}$, and productivity of $3.29 \mathrm{~g} / \mathrm{L} / \mathrm{h}$ (Table 1 ). As previously illustrated, the bacterial growth was inhibited by peroxides (at $\mu \mathrm{M})$ occurring in crude glycerol [34]. Tert-butyl hydroperoxide (TBHP) was identified as the major compound contributing to the cytotoxicity. The effect of TBHP on strain EcoB-170 was then investigated by culturing the strain with $20 \mathrm{~g} / \mathrm{L}$ pure glycerol plus TBHP ranging 60-90 $\mu \mathrm{M}$. Consequently, the cell growth remained unaffected. Another study reported that the lactic acid-iron complex mediated the generation of the reactive hydroxyl radical (HO) from peroxides [35]. Taken together, strain 
Table 1 D-Lactate production by microbes utilizing glycerol

\begin{tabular}{|c|c|c|c|c|c|c|}
\hline Strain & Operation & Substrate & Titer (g/L) & Yield (g/g) & Productivity (g/L/h) & References \\
\hline \multicolumn{7}{|l|}{ E. coli } \\
\hline EcoB-140B & Batch & Crude glycerol & $100^{b}$ & $0.95^{c}$ & $1.85^{d}$ & This study \\
\hline EcoB-170 & Two-dose ${ }^{a}$ & Crude glycerol & $115^{\mathrm{b}}$ & $0.97^{c}$ & $3.29^{d}$ & This study \\
\hline BLac-2106 & Fed-batch & Crude glycerol & 105 & 0.87 & 2.63 & [15] \\
\hline$A C-521$ & Fed-batch & Pure glycerol & 86 & 0.88 & 0.97 & {$[28]$} \\
\hline B0013-070 & Fed-batch & Crude glycerol & 100 & 0.75 & 2.78 & {$[26]$} \\
\hline B0013-080A & Fed-batch & Crude glycerol & 120 & 0.87 & 4.11 & {$[27]$} \\
\hline \multicolumn{7}{|c|}{ Klebsiella pneumoniae } \\
\hline ATCC25955 & Fed-batch & Pure glycerol & 142 & 0.82 & 2.96 & {$[28]$} \\
\hline
\end{tabular}

a The batch fermentation was conducted with the administration of two substrate doses

b The measured titer was the apparent concentration of D-lactate in the fermentation broth

c The yield based on crude glycerol was calculated by multiplication of the measured titer with the culture volume plus the added volume of the neutralizing solution

d Productivity was calculated by dividing the measured titer with the whole fermentation time (time zero to the end of the fermentation)

EcoB-170 is likely evolved to tolerate the induced oxidative stress. Nevertheless, the impurities of crude glycerol vary with various feedstock origins. There is need for further investigation of the detailed mechanism which underlines the resistance of the strain to crude glycerol.

\section{Conclusions}

In conclusion, this study proposed a simple fermentation technology. This fermentation strategy can be easily operated without the DO control. The result of this approach achieves the highest yield based on glycerol and a comparable titer and productivity as compared to those by fed-batch fermentation (Table 1). This proposed technology holds a promise to sustain the continued development of the crude glycerol-based biorefinery.

\section{Methods}

\section{Strain construction and adaptive evolution}

To enhance the expression level, endogenous gldA, dhaKLM, or $l d h A$ was fused with $\mathrm{P} \lambda \mathrm{P}_{\mathrm{L}}$. This construction work essentially followed the reported protocol. In brief, the $\mathrm{LE}^{*}$-gen-RE*-P $\lambda \mathrm{P}_{\mathrm{L}}$ cassette flanked by two homologous extensions was amplified from plasmid pPL-Gn by PCR with Gld1-Gld2 or Dha1-Dha2 primers [15]. By the act of $\lambda$ Red, the genomic locus was targeted by recombination with the electroporated PCR DNA in the host strain. The integrant strain was scored after removal of the inserted marker (i.e., gen) using Cre [36]. To delete $\operatorname{pox} B$, the truncated gene was amplified from strain JW2121-1 ( $\triangle$ poxB::FRT-kan-FRT) by PCR with the primers (cgctgaaggttacgtactgg and tagtcctgcagagcattaacg). The $\lambda$ Red-mediated recombination resulted in the integration of the PCR DNA into the genome of the strain, and the kan marker flanked by FRT was removed using Flp [37].
Crude glycerol was provided by Great Green Technology (Changhua, Taiwan). Adaptive evolution of the strain was carried out by a serial of cell subculture with the increasing level of crude glycerol. In general, the tobe-evolved strain was cultured in the Erlenmeyer flask $(125 \mathrm{~mL})$ containing NBS medium $(20 \mathrm{~mL})$ plus the indicated amount of crude glycerol with or without yeast extract. The bacterial culture was incubated at $37^{\circ} \mathrm{C}$ with vigorous shaking for $24 \mathrm{~h}$, and the cell density was measured turbidimetrically at $550 \mathrm{~nm}\left(\mathrm{OD}_{550}\right)$. Cell subculture was prepared by transferring the adapted culture to a flask containing the fresh medium. The cell subculture was repeated as needed.

\section{Bacterial fermentation}

Batch fermentation was performed as follows. The overnight culture was routinely prepared with a flask containing Luria-Bertani (LB) medium [38]. The experiment was conducted by inoculating the overnight culture into flasks containing $100 \mathrm{~mL}$ NBS medium [15] with $20 \mathrm{~g} / \mathrm{L}$ crude glycerol. The seeding culture was incubated at $37{ }^{\circ} \mathrm{C}$ with vigorous shaking for $12 \mathrm{~h}$. Bacteria were harvested by centrifugation and inoculated into a bioreactor (New Brunswick Bioflow 110) containing 1-L NBS medium plus crude glycerol and yeast extract as indicated. The initial cell density was maintained at $\mathrm{OD}_{550}$ of 1 or 0.2 . The operation condition was set up as follows unless stated otherwise. In the growth phase, the DO of the fermentation broth was maintained at $30 \%$ of the saturated level by purging air at $1 \mathrm{vvm}$ and the automatic control of the agitation rate. In the production phase, the DO was controlled at 3\% of the saturated level throughout the fermentation. Alternatively, crude glycerol $(80-90 \mathrm{~g} / \mathrm{L})$ was added into the culture broth and the fermentation was performed without controlling the DO. 
The fermentation was conducted at $37{ }^{\circ} \mathrm{C}$ and the broth $\mathrm{pH}$ was maintained at 7 using $20 \%(\mathrm{w} / \mathrm{v}) \mathrm{Ca}(\mathrm{OH})_{2}$.

\section{Enzyme assay}

The enzyme activity of lactate dehydrogenase was determined based on the previous report [15]. The cell-free extract was prepared and added to the reaction solution consisting of 1-mM pyruvate, $0.053-\mathrm{mM} \mathrm{NADH}$, and 100-mM sodium phosphate buffer ( $\mathrm{pH}$ 7.0). The reaction was carried out at $25{ }^{\circ} \mathrm{C}$ and the absorbance at $340 \mathrm{~nm}$ was measured along the time course. The enzyme activity was calculated according to the reduction in the NADH level in $\mu$ mol per min.

Following the reported protocol [15], the expression level of gldA and $d h a K$ was quantified by calculation of the individual mRNA level normalized to that of $i h f B$. In brief, total RNAs of the strain were isolated by Ambion ${ }^{\circledR}$ RiboPure (Life technology, USA) and used to synthesize the corresponding cDNAs with High Capacity cDNA Reverse Transcription kit (Life technology), the Power SYBR $^{\circledR}$ Green PCR Master Mix and Power SYBR ${ }^{\circledR}$ Green RT-PCR Reagent kit. The real-time PCR analysis was carried out using the Applied Biosystems ${ }^{\circledR}$ StepOne RealTime PCR System (Life technology).

\section{Fermentation analysis}

High-Performance Liquid Chromatography AS3000 (Thermo Scientific, USA) was used for the fermentation analysis. Glycerol was determined with the ICSep ICE-ION-300 column (Transgenomic, USA) and a RI detector. Organic acids were measured with the ICSep ICE-ORH-801 column (Transgenomic, USA) and a UV detector set at $210 \mathrm{~nm}$. The mobile phase consisting of $0.0085 \mathrm{~N} \mathrm{H}_{2} \mathrm{SO}_{4}$ was used for the analysis. Analysis methods essentially followed the previous report [15].

\section{Supplementary information}

Supplementary information accompanies this paper at https://doi. org/10.1186/s13068-019-1615-4.

Additional file 1: Figure S1. The time course of the E. coli strain undergoing adaptive evolution.

\section{Abbreviations}

a-KG: a-ketoglutarate; DO: dissolved oxygen; LAB: lactic acid bacteria; LDH: lactate dehydrogenase; $P \lambda P_{L}: \lambda P_{L}$ promoter; LB: Luria-Bertani; OAA: oxaloacetate; PLA: polylactide; TCA: tricarboxylic acid; TBHP: tert-butyl hydroperoxide; VGHF: very high gravity fermentation.

\section{Acknowledgements}

We like to acknowledge Dr. Wang ZW for the technical support.

\section{Authors' contributions}

YDW constructed bacterial strains and performed fermentation; JYL performed fermentation; CJC and YPC assisted in the design of the study and drafted the manuscript. All authors read and approved the final manuscript.

\section{Funding}

This work is supported by Ministry of Science and Technology (MOST 105-2221-E-035-085-MY3), Taiwan, and China Medical University (CMU105-S-50).

\section{Availability of supporting data \\ Not applicable.}

Ethics approval and consent to participate Not applicable.

\section{Consent for publication}

All authors agree to submit the work to the journal.

\section{Competing interests}

The authors declare that they have no competing interests.

\section{Author details}

1 Department of Chemical Engineering, Feng Chia University, 100 Wenhwa Road, Taichung 40724, Taiwan. ${ }^{2}$ Department of Medical Laboratory Science and Biotechnology, China Medical University, No. 91, Hsueh-Shih Road, Taichung 40402, Taiwan. ${ }^{3}$ Department of Medical Research, China Medical University Hospital, Taichung 40447, Taiwan. ${ }^{4}$ Department of Health and Nutrition Biotechnology, Asia University, Taichung 41354, Taiwan.

Received: 13 Auqust 2019 Accepted: 13 November 2019

Published online: 20 November 2019

References

1. Qi X, Ren Y, Wang X. New advances in the biodegradation of Poly(lactic) acid. Int Biodeterior Biodegr. 2017;117:215-23.

2. Farah S, Anderson DG, Langer R. Physical and mechanical properties of PLA, and their functions in widespread applications - a comprehensive review. Adv Drug Deliv Rev. 2016;107:367-92.

3. Wee YJ, Kim JN, Ryu HW. Biotechnological production of lactic acid and its recent applications. Food Technol Biotechnol. 2006;44:163-72.

4. Ikada Y, Jamshidi K, Tsuji H, Hyon SH. Stereocomplex formation between enantiomeric poly(lactides). Macromolecules. 1987;20:904-6.

5. Garlotta D. A literature review of poly(lactic acid). J Polym Environ. 2001;9:63-84.

6. Abdel-Rahmana MA, Tashiro Y, Sonomoto K. Recent advances in lactic acid production by microbial fermentation processes. Biotechnol Adv. 2013:31:877-902.

7. Hofvendahl K, Hahn-Hägerdal B. Factors affecting the fermentative lactic acid production from renewable resources. Enzyme Microbiol Technol. 2000;26:87-107.

8. Litchfield JH. Lactic acid, microbially produced. In: Schaechter MO, editor. Encyclopedia of microbiology. Oxford: Academic Press; 2009. p. 362-72.

9. Assavasirijinda N, Ge D, Yu B, Xue Y, Ma Y. Efficient fermentative production of polymer-grade D-lactate by an engineered alkaliphilic Bacillus sp. strain under non-sterile conditions. Microb Cell Fact. 2016;15:3.

10. Zhou L, Niu DD, Tian KM, Chen XZ, Prior BA, Shen W, Shi GY, Singh S, Wang ZX. Genetically switched D-lactate production in Escherichia coli. Metab Eng. 2012;14:560-8.

11. Tsuge $Y$, Yamamoto $S$, Kato N, Suda M, Vertès AA, Yukawa H, Inui M. Overexpression of the phosphofructokinase encoding gene is crucial for achieving high production of D-lactate in Corynebacterium glutamicum under oxygen deprivation. Appl Microbiol Biotechnol. 2015;99:4679-89.

12. Klotz S, Kaufmann N, Kuenz A, Prüße U. Biotechnological production of enantiomerically pure D-lactic acid. Appl Microbiol Biotechnol. 2016:100:9423-37.

13. da Silva GP, Mack M, Contiero J. Glycerol: a promising and abundant carbon source for industrial microbiology. Biotechnol Adv. 2009;27:30-9. 
14. Yang F, Hanna MA, Sun R. Value-added uses for crude glycerol-a byproduct of biodiesel production. Biotechnol Biofuels. 2012;5:1-13.

15. Wang ZW, Saini M, Lin LJ, Chiang CJ, Chao YP. Systematic engineering of Escherichia coli for D-lactate production from crude glycerol. J Agric Food Chem. 2015;63:9583-9.

16. Tarmy EM, Kaplan NO. Kinetics of Escherichia coli B D-lactate dehydrogenase and evidence for pyruvate-controlled change in conformation. J Biol Chem. 1968;243:2587-96.

17. Durnin G, Clomburg J, Yeates Z, Alvarez PJ, Zygourakis K, Campbell P, Gonzalez R. Understanding and harnessing the microaerobic metabolism of glycerol in Escherichia coli. Biotechnol Bioeng. 2009;103:148-61.

18. Martínez-Gómez K, Flores N, Castañeda HM, Martínez-Batallar G, Hernández-Chávez G, Ramírez OT, Gosset G, Encarnación S, Bolivar F. New insights into Escherichia coli metabolism: carbon scavenging, acetate metabolism and carbon recycling responses during growth on glycerol. Microb Cell Fact. 2012;11:46.

19. Chatzifragkou A, Papanikolaou S. Effect of impurities in biodiesel-derived waste glycerol on the performance and feasibility of biotechnological processes. Appl Microbiol Biotechnol. 2012;95:13-27.

20. Spiro S, Guest JR. Adaptive responses to oxygen limitation in Escherichia coli. Trends Biochem Sci. 1991;16:310-4.

21. Kim BH, Gadd GM. Bacterial physiology and metabolism. New York: Cambridge University Press; 2008.

22. Gennis RB, Stewart V. Respiration. In: Neidhardt FC, Curtiss III R, Ingraham JL, Lin ECC, Low KB, Magasanik B, ReznikoffWS, Riley M, Schaechter $\mathrm{M}$, Umbarger HE, editors. Escherichia coli and Salmonella, cellular and molecular biology. Washington, DC: ASM Press; 1996. p. 217-61.

23. Tran $\mathrm{QH}$, Bongaerts J, Vlad D, Unden G. Requirement for the protonpumping NADH dehydrogenase I of Escherichia coli in respiration of $\mathrm{NADH}$ to fumarate and its bioenergetic implications. Eur J Biochem. 1997:244:155-60.

24. Abdel-Hamid AM, Attwood MM, Guest JR. Pyruvate oxidase contributes to the aerobic growth efficiency of Escherichia coli. Microbiology. 2001;147:1483-98.

25. Mather MW, Gennis RB. Kinetic studies of the lipid-activated pyruvate oxidase flavoprotein of Escherichia coli. J Biol Chem. 1985;260:16148-55.

26. Chen XZ, Tian KM, Niu DD, Shen W, Algasan G, Singh S, Wang ZX. Efficient bioconversion of crude glycerol from biodiesel to optically pure D-lactate by metabolically engineered Escherichia coli. Green Chem. 2014;16:342-50.

27. Tian K, Niu D, Liu X, Prior BA, Zhou L, Lu F, Singh S, Wang Z. Limitation of thiamine pyrophosphate supply to growing Escherichia coli switches metabolism to efficient D-lactate formation. Biotechnol Bioeng. 2016;113:182-8.

28. Hong AA, Cheng KK, Peng F, Zhou S, Sun Y, Liu CM, Liu DH. Strain isolation and optimization of process parameters for bioconversion of glycerol to lactic acid. J Chem Technol Biotechnol. 2009;84:1576-81.

29. Feng $X$, Ding $Y$, Xian M, Xu X, Zhang R, Zhao G. Production of optically pure $\mathrm{D}$-lactate from glycerol by engineered Klebsiella pneumoniae strain. Bioresour Technol. 2014;172:269-75.

30. Puligundla P, Smogrovicova D, Obulam VSR, Ko S. Very high gravity (VHG) ethanolic brewing and fermentation: a research update. J Ind Microbiol Biotechnol. 2011;38:1133-44.

31. Thomas K, Ingledew WJ. Production of $21 \%(\mathrm{~V} / \mathrm{V})$ ethanol by fermentation of very high gravity (VHG) wheat mashes. J Ind Microbiol. 1992;10:61-8.

32. Rossi FG, Ribeiro MZ, Converti A, Vitolo M, Pessoa A. Kinetic and thermo dynamic aspects of glucose-6-phosphate dehydrogenase activity and synthesis. Enzyme Microb Technol. 2003;32:107-13.

33. Magdouli S, Saffar T, Guedri T, Tarek R, Brar SK, Blais JF. Practical aspects and case studies of industrial scale fermentation. In: Brar SK, Das PK Sarma SJ, editors. Microbial sensing in fermentation. Wiley-Blackwell: New Jersey; 2018. p. 267-98.

34. Nguyen ADQ, Kim YG, Kim SB, Kim CJ. Improved tolerance of recombinant Escherichia coli to the toxicity of crude glycerol by overexpressing trehalose biosynthetic genes (otsBA) for the production of $\beta$-carotene. Bioresour Technol. 2013;143:531-7.

35. Bruno-Barcena JM, Azcarate-Peril MA, Hassan HM. Role of antioxidant enzymes in bacterial resistance to organic acids. Appl Environ Microbiol. 2010:76:2747-53.

36. Chiang CJ, Saini M, Lee HM, Wang ZW, Lin LJ, Chao YP. Genomic engineering of Escherichia coli by the phage attachment site-based integration system with mutant loxP sites. Proc Biochem. 2012;17:2246-54.

37. Chiang CJ, Chen PT, Chao YP. Replicon-free and markerless methods for genomic insertion of DNAs in phage attachment sites and controlled expression of chromosomal genes in Escherichia coli. Biotechnol Bioeng. 2008;101:985-95.

38. Miller JH. Experiments in molecular genetics. Cold Spring Harbor Laboratory: Cold Spring Harbor; 1972.

\section{Publisher's Note}

Springer Nature remains neutral with regard to jurisdictional claims in published maps and institutional affiliations.
Ready to submit your research? Choose BMC and benefit from:

- fast, convenient online submission

- thorough peer review by experienced researchers in your field

- rapid publication on acceptance

- support for research data, including large and complex data types

- gold Open Access which fosters wider collaboration and increased citations

- maximum visibility for your research: over 100M website views per year

At BMC, research is always in progress.

Learn more biomedcentral.com/submissions 